\title{
THE RATIO OF RETROGRADE TO PROGRADE ORBITS: A TEST FOR KUIPER BELT BINARY FORMATION THEORIES
}

\author{
Hilke E. Schlichting ${ }^{1}$ and Re'em SARI ${ }^{1,2}$ \\ Received 2008 February 29; accepted 2008 June 12
}

\begin{abstract}
With the discovery of Kuiper Belt binaries that have wide separations and roughly equal masses, new theories were proposed to explain their formation. Two formation scenarios were suggested by Goldreich and collaborators. In the first, dynamical friction generated by a sea of small bodies enables a transient binary to become bound (the $\mathrm{L}^{2} \mathrm{~s}$ mechanism); in the second, a transient binary gets bound by an encounter with a third body (the $\mathrm{L}^{3}$ mechanism). We show that these different binary formation scenarios leave their own unique signatures in the relative abundance of prograde to retrograde binary orbits. This signature is due to the fact that stable retrograde orbits can exist much further out in the Hill sphere than prograde orbits. This provides an excellent opportunity to distinguish between the different binary formation scenarios observationally. We predict that if binary formation proceeded while sub-Hill velocities prevailed, the vast majority of all binaries with comparable masses would have retrograde orbits. This dominance of retrograde binary orbits is a result of binary formation via the $\mathrm{L}^{2} \mathrm{~s}$ mechanism, or any other mechanism that dissipates energy in a smooth and gradual manner. For super-Hill velocities, binary formation proceeds via the $\mathrm{L}^{3}$ mechanism, which produces a roughly equal number of prograde and retrograde binaries. These predictions assume that subsequent orbital evolution due to dynamical friction and dynamical stirring of the Kuiper Belt did not alter the sense of the binary orbit after formation.
\end{abstract}

Subject headings: Kuiper Belt — planets and satellites: formation

\section{INTRODUCTION}

The detection of binaries with comparable masses and wide separations in the Kuiper Belt called for new theories to explain their formation (e.g., Weidenschilling 2002; Goldreich et al. 2002; Funato et al. 2004; Astakhov et al. 2005; Lee et al. 2007). Their existence cannot be explained with a formation scenario that involves a collision and tidal evolution, as has been proposed for the formation of the Moon and Charon (Hartmann \& Davis 1975; Cameron \& Ward 1976; McKinnon 1989), since it cannot account for the current angular momentum of the binary system. In a formation scenario proposed by Weidenschilling (2002), two Kuiper Belt objects (KBOs) collide with each other inside the Hill sphere of a third. However, in the Kuiper Belt, gravitational scattering between the two intruders is about 100 times $^{3}$ more common than a collision. Therefore, three-body gravitational deflection (the $\mathrm{L}^{3}$ mechanism), as proposed by Goldreich et al. (2002), should dominate the binary formation over such a collisional scenario. A second binary formation scenario suggested by Goldreich et al. (2002), called the $\mathrm{L}^{2} \mathrm{~s}$ mechanism, consists of the formation of a transient binary that gets bound with the aid of dynamical friction from a sea of small bodies. In the formation scenario of Astakhov et al. (2005) and Lee et al. (2007), the existence of longlived transient binaries that spend a long time in their mutual Hill sphere, near a periodic orbit, is responsible for the creation of Kuiper Belt binaries (KBBs). Finally, Funato et al. (2004) proposed a binary formation mechanism that involves a collision between two large KBOs. This collision creates a small moon that is replaced in an exchange reaction by a massive body with a high eccentricity and a large semimajor axis.

\footnotetext{
1 Theoretical Astrophysics, California Institute of Technology, MC 130-33, Pasadena, CA 91125; hes@astro.caltech.edu, sari@tapir.caltech.edu.

2 Racah Institute of Physics, Hebrew University, Jerusalem 91904, Israel.

3 For this estimate we used a value of $\alpha \sim 10^{-4}$ and assumed that the velocity dispersion of the KBOs at the time of binary formation was less than their Hill velocity; see $\S 2$ for details.
}

In this paper, we show that the $\mathrm{L}^{2} \mathrm{~s}$ and $\mathrm{L}^{3}$ mechanisms leave unique signatures in the relative abundance of prograde to retrograde binary orbits. The $\mathrm{L}^{2} \mathrm{~s}$ mechanism dominates over the $\mathrm{L}^{3}$ mechanism for sub-Hill velocities (Schlichting \& Sari 2008). We argue that binaries formed from dynamically cold KBOs by the $\mathrm{L}^{2} \mathrm{~S}$ mechanism have retrograde orbits. This is due to the existence of stable retrograde binary orbits with modified Jacobi constants similar to those of unbound KBOs on circular orbits that have impact parameters that correspond to distances of closest approach of less than the Hill radius. No equivalent prograde orbits exist (e.g., Hénon 1970; Innanen 1979; Zhang \& Innanen 1988; Hamilton \& Burns 1991; Hamilton \& Krivov 1997). Since dynamical friction only gradually increases the modified Jacobi constant (for a binary, this corresponds to gradually increasing the absolute value of the binding energy), all binaries that form via the $\mathrm{L}^{2} \mathrm{~s}$ mechanism, or any other mechanism that dissipates energy in a smooth and gradual manner, will start with modified Jacobi constants that are close to those of unbound KBOs that penetrate the Hill sphere and hence have retrograde orbits. For super-Hill KBO velocities, only the $\mathrm{L}^{3}$ mechanism can form tight binaries that tend to survive (Schlichting \& Sari 2008). The fact that retrograde orbits are stable for larger semimajor axes is no longer of importance, since only tight binaries are saved from breakup. This therefore leads to the formation of a roughly equal number of prograde and retrograde binaries for super-Hill KBO velocities.

Our paper is structured as follows. In $\S 2$ we outline our assumptions, explain our choice of parameters, and define variables that will be used throughout this paper. We calculate the ratio of prograde to retrograde binary orbits for the $\mathrm{L}^{2} \mathrm{~s}$ and $\mathrm{L}^{3}$ mechanisms and predict the relative abundance of prograde to retrograde orbits for sub-Hill and super-Hill KBO velocities in $\S 3$. We compare our predictions with observations in $\S 4$. Discussion and conclusions follow in $\S 5$.

\section{DEFINITIONS AND ASSUMPTIONS}

The Hill radius denotes the distance from a body at which the tidal forces due to the Sun and the gravitational force due to the 
body, both acting on a test particle, are in equilibrium. It is given by

$$
R_{\mathrm{H}} \equiv a_{\odot}\left(\frac{m_{1}+m_{2}}{3 M_{\odot}}\right)^{1 / 3}
$$

where $m_{1}$ and $m_{2}$ are the masses of the two KBOs, $a_{\odot}$ is their semimajor axis around the Sun, and $M_{\odot}$ is the mass of the Sun. Our definition of the Hill radius differs from that used by Schlichting $\&$ Sari (2008), since we include the combined mass of both KBOs here. We chose to do so since it will make comparisons with works by other authors easier.

We use the "two-group approximation" (Goldreich et al. 2002, 2004), which consists of the identification of two groups of objects: small ones, which contain most of the total mass, with surface mass density $\sigma$; and large ones, which contain only a small fraction of the total mass, with surface mass density $\Sigma \ll \sigma$. We assume that $\sigma \sim 0.3 \mathrm{~g} \mathrm{~cm}^{-2}$, which is the extrapolation of the minimum-mass solar nebula (Hayashi 1981) to a heliocentric distance of 40 AU. Estimates from Kuiper Belt surveys (Trujillo et al. 2001; Trujillo \& Brown 2003; Petit et al. 2008a; Fraser et al. 2008; Fuentes \& Holman 2008) yield a value of $\Sigma \sim 3 \times 10^{-4} \mathrm{~g} \mathrm{~cm}^{-2}$ for KBOs with radii of $R \sim 100 \mathrm{~km}$. We use this value of $\Sigma$, assuming that the value of $\Sigma$ during the formation of KBBs was the same as it is now. Our choices for the values of $\Sigma$ and $\sigma$ are also consistent with results from numerical coagulation simulations by Kenyon \& Luu (1999).

Large bodies grow by the accretion of small bodies. Large KBOs viscously stir the small bodies, increasing the small bodies' velocity dispersion $u$. As a result, $u$ grows on the same timescale as $R$, provided that mutual collisions among the small bodies are not yet important. In this case, $u$ is given by

$$
\frac{u}{v_{\mathrm{H}}} \sim\left(\frac{\Sigma}{\sigma \alpha}\right)^{1 / 2} \sim 3
$$

where $\alpha=R / R_{\mathrm{H}} \sim 10^{-4}$ at $40 \mathrm{AU}$ (Goldreich et al. 2002) and $v_{\mathrm{H}}$ is the Hill velocity of the large bodies, which is given by $v_{\mathrm{H}}=$ $\Omega R_{\mathrm{H}}$, where $\Omega$ is the orbital frequency around the Sun. The velocity $v$ of the large KBOs increases due to mutual viscous stirring, but is damped by dynamical friction from a sea of small bodies such that $v<u$. Balancing the stirring and damping rates of $v$ and substituting for $u$ from equation (2), we find that

$$
\frac{v}{v_{\mathrm{H}}} \sim \alpha^{-2}\left(\frac{\Sigma}{\sigma}\right)^{3} \sim 0.1 .
$$

For our choice of parameters, we have sub-Hill KBO velocities during the epoch of formation of bodies with $R \sim 100 \mathrm{~km}$. We therefore focus our work on the shear-dominated velocity regime $\left(v \ll v_{\mathrm{H}}\right)$. However, we also discuss how our results would be modified if $v \gg v_{\mathrm{H}}$.

\section{PROGRADE VERSUS RETROGRADE BINARY ORBITS}

\subsection{Sub-Hill Velocities: $v \ll v_{\mathrm{H}}$}

The disk of KBOs is effectively two-dimensional in the sheardominated velocity regime $\left(v \ll v_{\mathrm{H}}\right)$, since the growth of their inclinations is suppressed (Wetherill \& Stewart 1993; Rafikov 2003; Goldreich et al. 2004). We therefore restrict our calculations for the shear-dominated velocity regime to two dimensions. Since we are interested in close encounters among the KBOs, their interaction is well described by Hill's equations (Hill 1878; Goldreich
\& Tremaine 1980; Hénon \& Petit 1986). In Hill coordinates, the equations of motion of the two KBOs can be decomposed into their center-of-mass motion and their relative motion with respect to one another. The modified Jacobi constant is exactly conserved in the Hill formalism, but the Hill formalism itself is an approximation to the general three-body problem. It assumes that the masses of bodies 1 and 2 (in our case, the two KBOs) are much less than that of the Sun. We use the standard Hill coordinate system and reference frame, as in Hénon \& Petit (1986) and Ida (1990). In this rotating frame, the direction of the $x$-axis is given by the line connecting the Sun and the center of mass of the two KBOs, such that the positive $x$-direction is pointing away from the Sun. The $y$-axis is perpendicular to the $x$-axis, pointing in the direction of the motion of the KBOs' center of mass around the Sun. In Hill coordinates, the modified Jacobi constant is

$$
J_{C}=3 x^{2}+\frac{6}{\left(x^{2}+y^{2}\right)^{1 / 2}}-\dot{x}^{2}-\dot{y}^{2},
$$

where $x$ and $y$ correspond to the relative separations between the two KBOs in the $x$ - and $y$-directions, respectively (Hénon \& Petit $1986)$. Length has been scaled by $R_{\mathrm{H}}$ and time by $\Omega^{-1}$. In Hill coordinates, the Lagrangian points $\mathrm{L} 1$ and $\mathrm{L} 2$ are located at $(-1,0)$ and $(+1,0)$, respectively, where we define $\mathrm{L} 1$ as the Lagrangian point located between the KBO and the Sun. Their modified Jacobi constants are $J_{C}(\mathrm{~L} 1)=J_{C}(\mathrm{~L} 2)=9$. From equation (4), we can see that tight binaries with small separations have values of $J_{C} \gg 9$. We call a binary orbit prograde if its angular momentum about the binary center of mass, as viewed in the nonrotating frame, is in the same direction as the orbital angular momentum of the binary around the Sun. If the binary angular momentum is in the opposite direction to the orbital angular momentum of the binary around the Sun, the orbit is called retrograde. Several authors recognized that planar retrograde orbits are stable for larger semimajor axes than prograde orbits (e.g., Hénon 1970; Innanen 1979; Zhang \& Innanen 1988; Hamilton \& Burns 1991; Hamilton \& Krivov 1997). A prograde binary with an initially circular orbit becomes unbound for values of $a \gtrsim 0.49 R_{\mathrm{H}}$, where $a$ is the initial semimajor axis of the mutual binary orbit (Hamilton \& Burns 1991). This implies that prograde orbits with modified Jacobi constants that are less than those of the Lagrangian points L1 and L2 are unbound. In contrast to the prograde case, there exist stable retrograde binary orbits that have values of $J_{C} \lesssim J_{C}(\mathrm{~L} 1)=$ $J_{C}(\mathrm{~L} 2)=9$. This result is also shown in Figure 1. Figure 1 shows histograms of $J_{C}$ for prograde and retrograde binaries formed by the $\mathrm{L}^{3}$ mechanism from KBOs with initially circular orbits around the Sun. In the remainder of this paper, we discuss the stability of prograde and retrograde orbits in terms of $J_{C}$ and not the semimajor axis, since the latter is not well defined (i.e., it is not a constant of motion) for wide orbits with $a \sim R_{\mathrm{H}}$. The modified Jacobi constant for two KBOs that approach each other from infinity is

$$
J_{C}=3 x^{2}-\dot{x}^{2}-\dot{y}^{2}=\frac{3}{4} b^{2}-e^{2},
$$

where $b$ is the initial separation between the two KBOs in the $x$-direction and $e$ is the relative eccentricity in Hill units, given by $\left|\boldsymbol{e}_{1}-\boldsymbol{e}_{2}\right|$, where $\boldsymbol{e}_{1}$ and $\boldsymbol{e}_{2}$ are the eccentricity vectors of body 1 and body 2 , respectively. Only KBOs with values of $b$ ranging from $1.7 R_{\mathrm{H}}$ to $2.5 R_{\mathrm{H}}$ penetrate each other's Hill spheres if started on circular orbits. From equation (5), we have therefore determined that only KBOs with values of $2.2 \leq J_{C} \leq 4.7$ have a distance of closest approach of $R_{\mathrm{H}}$ or less, provided that they started on circular orbits around the Sun. 


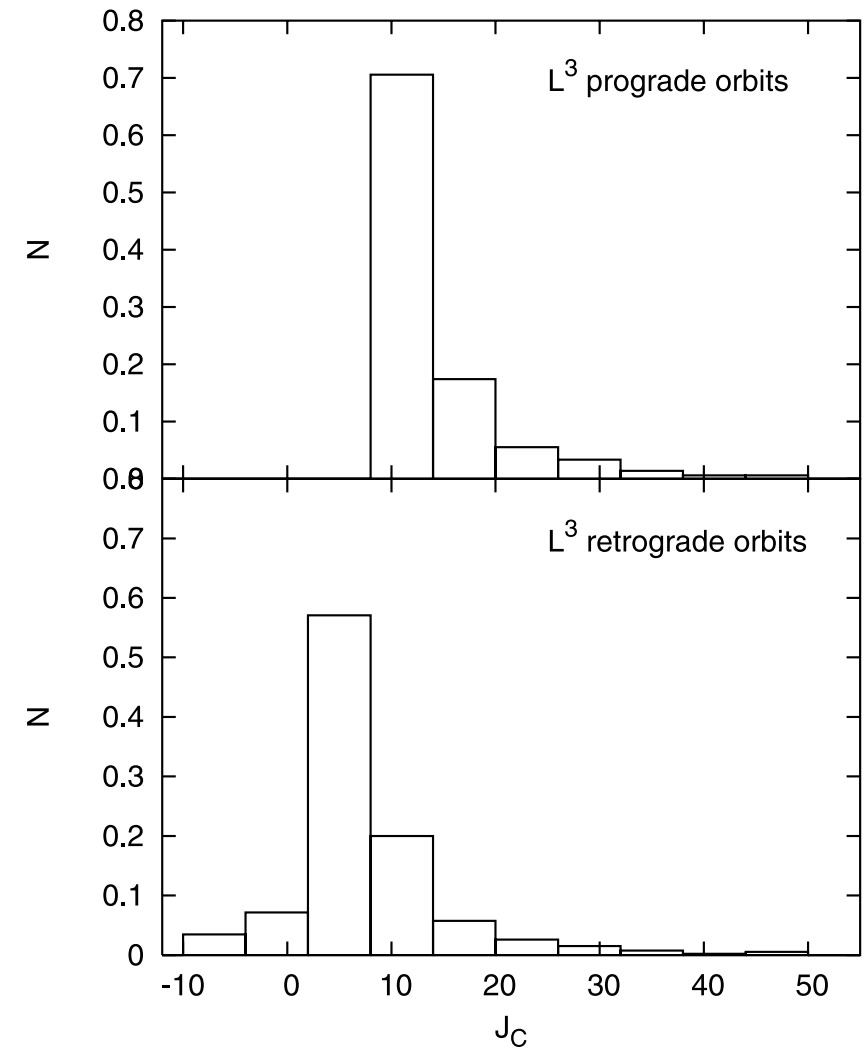

FIG. 1.- Histograms of the modified Jacobi constants, $J_{C}$, of prograde and retrograde $\mathrm{KBB}$ s that formed via three-body gravitational deflection, the $\mathrm{L}^{3}$ mechanism, for $v \ll v_{\mathrm{H}}$. Each histogram is normalized to unity, but overall, the retrograde orbits are twice as abundant as the prograde orbits. Note that prograde binaries exist only for values of $J_{C} \gtrsim 9$, whereas retrograde binaries also exist for values of $J_{C} \lesssim 9$.

\subsection{1. $L^{2}$ s Mechanism}

In the $\mathrm{L}^{2} \mathrm{~s}$ mechanism, KBBs form from transient binaries that become bound with the aid of dynamical friction from a sea of small bodies. This dynamical friction provides a gentle force that damps the random velocity of large KBOs. For typical parameters, the dynamical friction force only extracts a small fraction of energy over an orbital timescale. Therefore, KBBs that form via the $\mathrm{L}^{2} \mathrm{~s}$ mechanism, or any other mechanism that dissipates energy gradually, have initially modified Jacobi constants similar to those of the unbound KBOs that penetrate within the Hill sphere. As mentioned above, for KBOs that started on circular orbits around the Sun, this corresponds to values of $2.2 \leq J_{C} \leq 4.7$. However, only stable retrograde orbits exist for values of $J_{C} \lesssim 9$. This implies that all KBBs that form this way must have retrograde orbits, since no stable prograde orbits exist for values of $J_{C} \lesssim 9$. Once a binary is formed, dynamical friction increases the modified Jacobi constant and the absolute value of the binary binding energy. We confirm that all binaries that form from KBOs on initially circular orbits around the Sun via the $\mathrm{L}^{2} \mathrm{~s}$ mechanism are retrograde by numerical integrations that are presented below.

Since it is not feasible to examine the interactions with each small body individually, their net effect is modeled by an averaged force that acts to damp the large KBOs' noncircular velocity around the Sun. We parameterize the strength of the damping by a dimensionless quantity $D$, defined as the fractional decrease in noncircular velocity due to dynamical friction over a time $\Omega^{-1}$ :

$$
D \sim \frac{\sigma}{\rho R}\left(\frac{u}{v_{\mathrm{H}}}\right)^{-4} \alpha^{-2} \sim \frac{\Sigma}{\rho R} \alpha^{-2}\left(\frac{v}{v_{\mathrm{H}}}\right)^{-1} .
$$

The first expression is simply an estimate of dynamical friction due to a sea of small bodies, in the regime in which $u>v_{\mathrm{H}}$. The second expression describes the mutual excitation among the large KBOs for values of $v \ll v_{\mathrm{H}}$. The velocity dispersion of the large KBOs, $v$, achieves a quasi-steady state on a timescale shorter than at which $R$ grows, since only a subset of the deflected bodies are accreted. The stirring among the large KBOs can therefore be equated to the damping due to dynamical friction (for a detailed derivation, see Goldreich et al. 2004).

Since the growth of the inclinations is suppressed in the sheardominated velocity regime, the disk of KBOs is effectively twodimensional (Wetherill \& Stewart 1993; Rafikov 2003; Goldreich et al. 2004). We therefore restrict this calculation to two dimensions. In Hill coordinates, the relative motion of two equal-mass KBOs, including the dynamical friction term, is governed by

$$
\begin{gathered}
\ddot{x}-2 \dot{y}-3 x=-\frac{3 x}{\left(x^{2}+y^{2}\right)^{3 / 2}}-D \dot{x}, \\
\ddot{y}+2 \dot{x}=-\frac{3 y}{\left(x^{2}+y^{2}\right)^{3 / 2}}-D(\dot{y}+1.5 x) .
\end{gathered}
$$

Length has been scaled by $R_{\mathrm{H}}$ and time by $\Omega^{-1}$. Equations (7) and (8) are integrated for different values of $D$ and impact parameters ranging from $1.7 R_{\mathrm{H}}$ to $2.5 R_{\mathrm{H}}$, with equal step size. Impact parameters outside this range result in a distance of closest approach between the two KBOs of more than $R_{\mathrm{H}}$.

For $D=0.01$, we performed 20,000 integrations. About $2 \%$ of these integrations resulted in the formation of a binary. Figure 2 shows three examples of the evolution of the specific angular momentum and $J_{C}$ of the binary formation events from our integrations for $D=0.01$. In addition, we performed integrations for values of $D$ ranging from 0.1 to 0.0004 and find that, just as in the $D=0.01$ case, only retrograde binaries form. We define $h$ as the specific angular momentum of the binary in the nonrotating frame. It can be written as $h=x \dot{y}-y \dot{x}+x^{2}+y^{2}$ and is related to the total binary orbital angular momentum, $L$, by $h=\left(1 / m_{1}+\right.$ $\left.1 / m_{2}\right) L$. The time $t=0$ corresponds to the time at which $y=0$ if the relative $\mathrm{KBO}$ velocity is solely due to the Keplerian shear (i.e., ignoring the actual gravitational interaction between the bodies). The evolution of $h$ and $J_{C}$ is shown until the binary separation has decreased to $0.1 R_{\mathrm{H}}$ or less. Binaries with separations of $0.1 R_{\mathrm{H}}$ or less are sufficiently tight that perturbations from the Sun are too weak to flip the sign of the angular momentum. As expected from our discussion above, the angular momenta of the binaries are negative, corresponding to retrograde binary orbits. In fact, all binaries that form via the $\mathrm{L}^{2} \mathrm{~s}$ mechanism in our numerical integrations display retrograde orbits. Dynamical friction shrinks the binary separation. As a result, the magnitude of the binary angular momenta decreases with time. The right-hand side of Figure 2 shows the evolution of the modified Jacobi constant. Newly formed binaries initially have a modified Jacobi constant of $<9$, which is possible only for retrograde binaries. Dynamical friction shrinks the semimajor axes of the binaries, which leads to an increase of $J_{C}$ with time while keeping the sense of the rotation, i.e., the sign of $h$, fixed. Eventually the modified Jacobi constant grows to values above $J_{C}(\mathrm{~L} 1)=J_{C}(\mathrm{~L} 2)=9$. For values of $J_{C} \gtrsim 9$, prograde orbits can exist; however, all binaries that formed with the aid of dynamical friction started out with values of $J_{C}<9$, for which only retrograde orbits are stable. Therefore, all KBBs that form via the $\mathrm{L}^{2} \mathrm{~s}$ mechanism, or any other mechanism that gradually removes energy from transient binaries, orbit each other in the retrograde sense, since otherwise they would not have been able to form in the first place. Figure 3 shows the evolution of $h$ and $J_{C}$ as a 

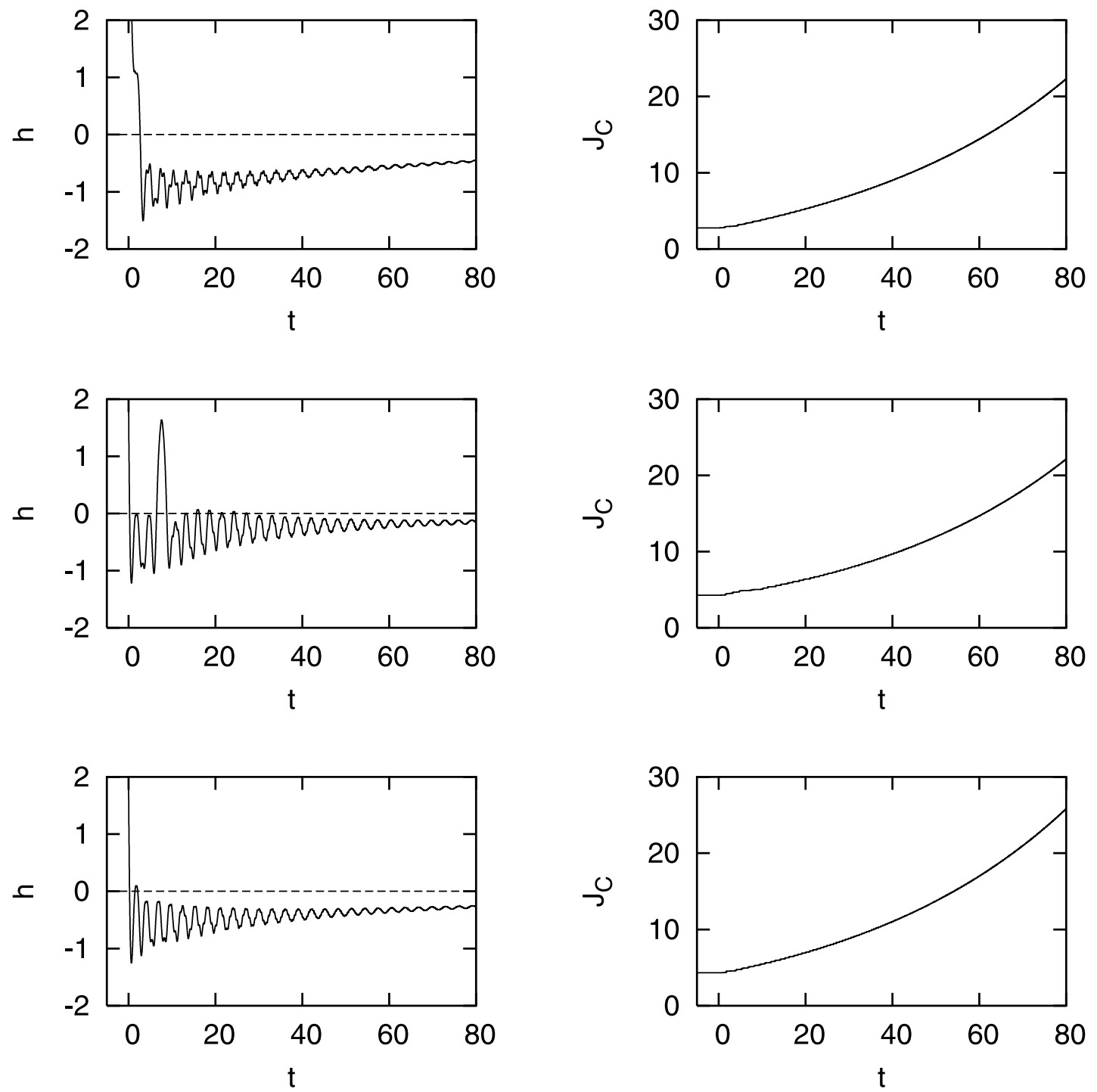

FIG. 2.- Three examples of $\mathrm{KBO}$ encounters in the $\mathrm{L}^{2} \mathrm{~s}$ mechanism for $v \ll v_{\mathrm{H}}$ and $D=0.01$ that result in the formation of a binary. The plots on the left- and righthand sides show the evolution of the specific angular momentum, $h$, and the modified Jacobi constant, $J_{C}$, respectively, as a function of time. The time $t=0$ corresponds to the time at which $y=0$ if the relative KBO velocity is solely due to the Keplerian shear (i.e., ignoring the actual gravitational interaction between the bodies). The evolution of $h$ and $J_{C}$ is shown until the binary separation has decreased to $0.1 R_{\mathrm{H}}$ or less. These examples show that the sense of rotation is practically preserved. The specific angular momentum, $h$, displays large variations right after capture that are caused by solar tides. The most extreme case of angular momentum sign change found in our simulations for bodies that form binaries is displayed in the second of the three examples. The angular momenta of the binaries are all negative, corresponding to retrograde binary orbits. In fact, all binaries that form via the $\mathrm{L}^{2} \mathrm{~s}$ mechanism in our numerical integrations display retrograde orbits. Dynamical friction shrinks the binary separation, leading to a decrease in the magnitude of $h$ and an increase of $J_{C}$ with time. The modified Jacobi constants of the newly formed binaries are smaller than $J_{C}(\mathrm{~L} 1)=9$, which explains why all their orbits are retrograde (see $\S 3.1 .1$ for details).

function of time for KBO encounters that did not lead to the formation of a binary. These examples show that KBOs encounter each other and leave each other with positive angular momenta. This is a result of the Keplerian shear and follows from the definition of $h$.

We have assumed here that all KBOs are initially on circular orbits around the Sun, and we have shown that this leads to the formation of exclusively retrograde binaries in the $\mathrm{L}^{2} \mathrm{~s}$ mechanism. If, however, the velocity dispersion of the KBOs is sufficiently large, such that $e$ is of the order of the Hill eccentricity, bigger impact parameters allow the KBOs to penetrate each other's Hill spheres. In this case, there now exist KBOs that have an initial value of $J_{C}$ just a little below 9 (see eq. [5]), in which case only a small change in $J_{C}$ is sufficient for the formation of retrograde and prograde binaries. Therefore, prograde binaries can form with the aid of dynamical friction, provided that the velocity dispersion of the KBOs is approximately $v_{\mathrm{H}}$.
Our prediction for the sense of the binary orbit relies on the assumption that dynamical friction does not alter the sense of the binary orbit in the subsequent binary evolution. Although we have shown in our simulations that for our dynamical friction model, this is indeed the case, the actual behavior of dynamical friction may differ from the model implemented here.

\subsection{2. $L^{3}$ Mechanism}

A transient binary forms when two large KBOs penetrate each other's Hill spheres. This transient binary must lose energy in order to become gravitationally bound. In the $\mathrm{L}^{3}$ mechanism, the excess energy is carried away by an encounter with a third massive body. This encounter can provide a significant change in energy, which corresponds to a considerable change in $J_{C}$. The modified Jacobi constants of KBBs that form via the $\mathrm{L}^{3}$ mechanism are therefore not constrained to values similar to those of their initial $J_{C}$; their orbits can therefore be either prograde or retrograde. We 

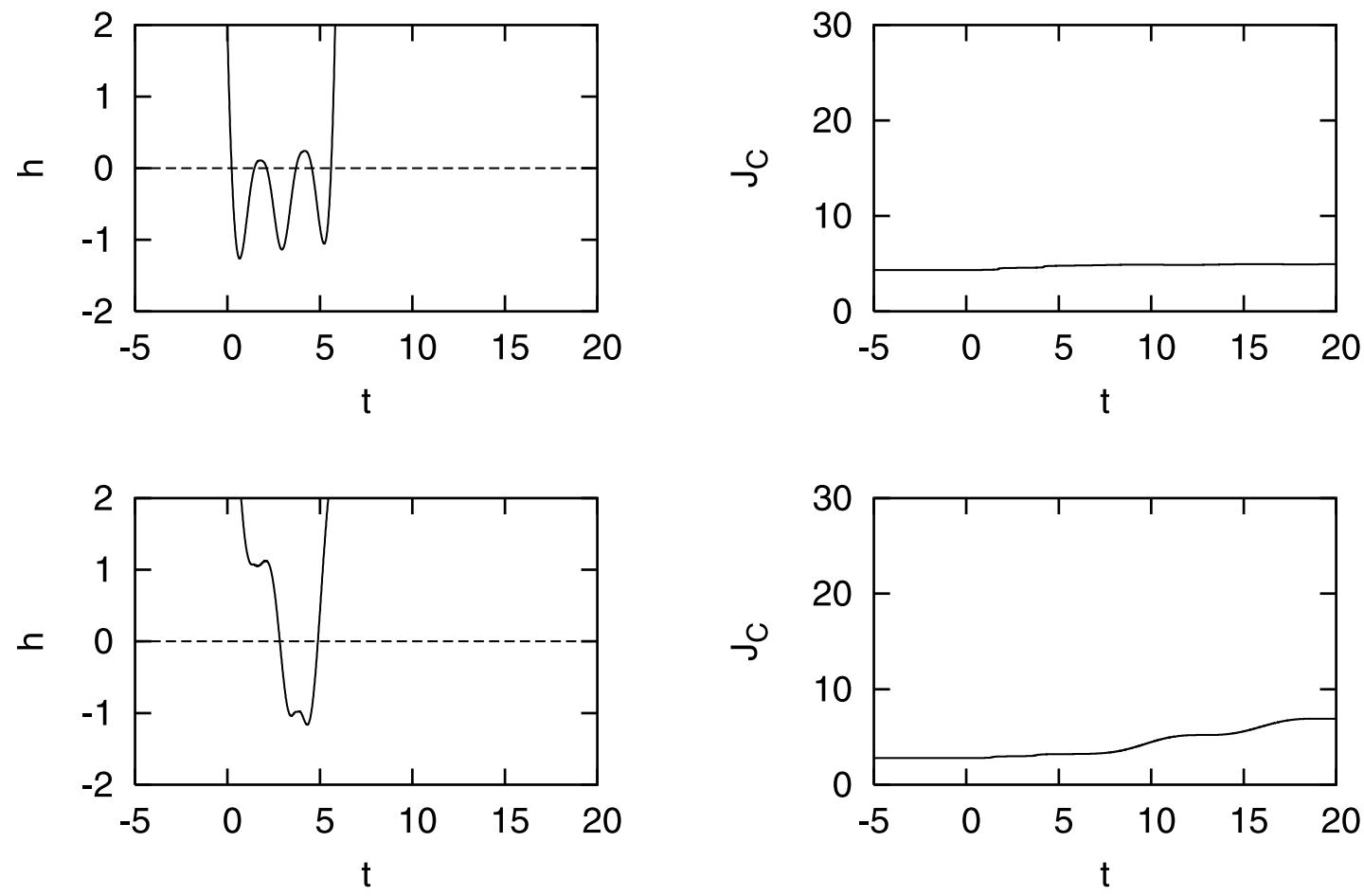

Fig. 3. - Same as in Fig. 2, but for two examples of KBO encounters in the $\mathrm{L}^{2} \mathrm{~s}$ mechanism with $v \ll v_{\mathrm{H}}$ and $D=0.01$ that do not result in the formation of a binary. As a result of the Keplerian shear, KBOs encounter and leave each other with positive values of $h$.

show that this is indeed the case with numerical integrations discussed below, and we determine the ratio of prograde to retrograde orbits for binary formation via the $\mathrm{L}^{3}$ mechanism.

Our calculation is performed in the shear-dominated velocity regime in two dimensions. As an initial condition, we assume that all bodies are on circular orbits. We modify Hill's equations (Hill 1878; Goldreich \& Tremaine 1980; Petit \& Hénon 1986) to include three equal-mass bodies besides the Sun. The equations of motion, with length scaled by $R_{\mathrm{H}}$ and time by $\Omega^{-1}$, for body 1 are given by

$$
\begin{aligned}
\ddot{x}_{1}-2 \dot{y}_{1}-3 x_{1}= & -\frac{3\left(x_{1}-x_{2}\right)}{2\left[\left(x_{1}-x_{2}\right)^{2}+\left(y_{1}-y_{2}\right)^{2}\right]^{3 / 2}} \\
& -\frac{3\left(x_{1}-x_{3}\right)}{2\left[\left(x_{1}-x_{3}\right)^{2}+\left(y_{1}-y_{3}\right)^{2}\right]^{3 / 2}}, \\
\ddot{y}_{1}+2 \dot{x}_{1}=- & \frac{3\left(y_{1}-y_{2}\right)}{2\left[\left(x_{1}-x_{2}\right)^{2}+\left(y_{1}-y_{2}\right)^{2}\right]^{3 / 2}} \\
& -\frac{3\left(y_{1}-y_{3}\right)}{2\left[\left(x_{1}-x_{3}\right)^{2}+\left(y_{1}-y_{3}\right)^{2}\right]^{3 / 2}} .
\end{aligned}
$$

The subscripts 1, 2, and 3 label the $x$ - and $y$-coordinates of KBOs 1 , 2 , and 3 , respectively. Similar equations of motion can be obtained for bodies 2 and 3 . The resulting binary orbits are calculated by numerically integrating the equations of motion. We refer the reader to Schlichting \& Sari (2008) for the exact details of these calculations.

Figure 1 shows histograms of the modified Jacobi constants of prograde and retrograde binaries that formed via the $\mathrm{L}^{3}$ mechanism. Both histograms are normalized to unity. As discussed above, we indeed find that prograde orbits only exist for values of $J_{C} \gtrsim 9$.
The stability of retrograde orbits extends below $J_{C}=9$, down to $J_{C} \sim-10$. It therefore includes the values of $J_{C}$ for circular heliocentric orbits that have a distance of closest approach of $R_{H}$ or less (i.e., $2.2<J_{C}<4.7$ ). Unlike the $\mathrm{L}^{2} \mathrm{~s}$ mechanism, the $\mathrm{L}^{3}$ mechanism does produce both retrograde and prograde binaries for $v \ll v_{\mathrm{H}}$. We find that $65 \%$ of all binary orbits are retrograde and $35 \%$ are prograde (see Fig. 4 ). Here, we only considered binary formation from three equal-mass bodies that started on initially circular orbits around the Sun. We therefore caution that

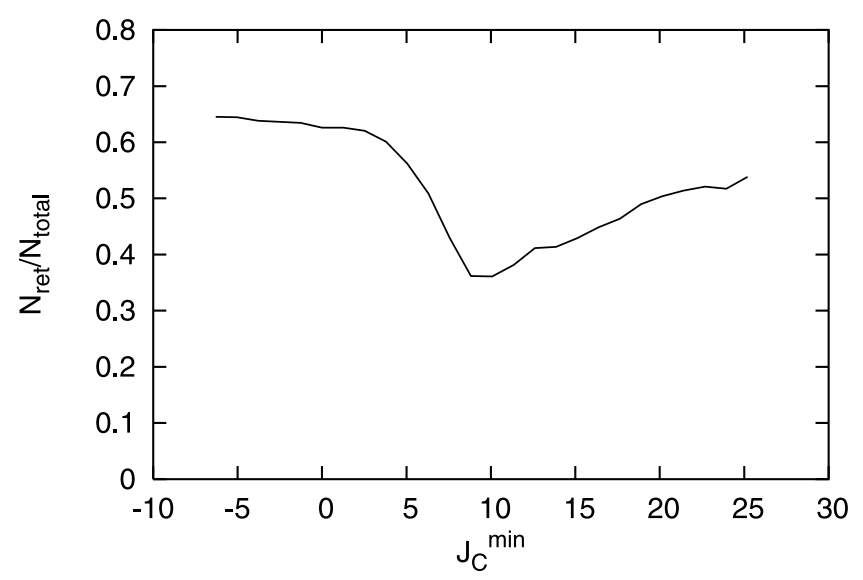

FIG. 4.- Ratio of retrograde binaries, $N_{\text {ret }}$, that have a modified Jacobi constant of $J_{C}^{\text {min }}$ or larger to the total number of binaries, $N_{\text {total }}$, that formed via the $\mathrm{L}^{3}$ mechanism for $v \ll v_{\mathrm{H}}$. For small values of $J_{C}^{\min }$, i.e., when all binaries are included, about 2/3 have retrograde orbits. More retrograde than prograde binaries form because retrograde binary orbits are stable further out in the Hill sphere than prograde ones. As $J_{C}^{\min }$ increases, the fraction of retrograde binaries decreases, reaching a minimum of about $1 / 3$ for $J_{C}^{\min } \sim 9$. This may be due to the Keplerian shear, which increases the duration of a prograde encounter between unbound $\mathrm{KBO}$ compared to a retrograde encounter. The fraction of prograde and retrograde binaries becomes comparable for $J_{C}^{\min } \gg 9$, because for such binaries, neither the Keplerian shear nor the increased stability of retrograde orbits are important. 
the ratio of prograde to retrograde orbits due to the $\mathrm{L}^{3}$ mechanism might differ for other mass ratios and velocity dispersions.

\subsubsection{The Ratio of Retrograde to Prograde Orbits}

Schlichting \& Sari (2008) have shown that for sub-Hill KBO velocities, the ratio of the $\mathrm{L}^{3}$ binary formation rate to the $\mathrm{L}^{2} \mathrm{~s}$ binary formation rate is

$$
\frac{\mathrm{FR}_{\mathrm{L}^{3}}}{\mathrm{FR}_{\mathrm{L}^{2} \mathrm{~s}}}=0.05 \frac{v}{v_{\mathrm{H}}} .
$$

Therefore, for sub-Hill KBO velocities, binaries in the Kuiper Belt form primarily because of dynamical friction. For our estimate of $v / v_{\mathrm{H}} \sim 0.1$, we find that $\mathrm{FR}_{\mathrm{L}^{3}} / \mathrm{FR}_{\mathrm{L}^{2} \mathrm{~s}} \sim 0.005$, in which case $\sim 0.5 \%$ of all binaries form directly by the $\mathrm{L}^{3}$ mechanism. Since prograde binaries can only form via the $\mathrm{L}^{3}$ mechanism, they make up a negligible fraction of the total binaries. Below we discuss how a somewhat larger fraction of prograde binaries can arise as a result of exchange reactions with unbound KBOs.

Once a binary is formed, its semimajor axis shrinks because of the dynamical friction provided by a sea of small bodies. Dynamical friction decreases the orbit of a KBB that has an orbital velocity $v_{B}$ at a rate of

$$
\mathcal{R}_{\mathrm{sh}} \sim D \Omega \sim \frac{\Sigma}{\rho R} \alpha^{-2}\left(\frac{v}{v_{\mathrm{H}}}\right)^{-1},
$$

where we assume that $v_{B}<u$. Exchange reactions or binary breakup due to passing KBOs occurs at a rate of

$$
\mathcal{R}_{\mathrm{ex}} \sim \frac{\Sigma}{\rho R} \alpha^{-2} \Omega\left(\frac{v_{B}}{v_{\mathrm{H}}}\right)^{-1}
$$

The ratio of these two rates is given by

$$
\frac{\mathcal{R}_{\mathrm{sh}}}{\mathcal{R}_{\mathrm{ex}}} \sim \frac{v_{B}}{v},
$$

where $v \ll v_{\mathrm{H}}$ and $v_{B} \gtrsim v_{\mathrm{H}}$. Breakup or exchange reactions are most likely for wide binaries, in which case $v_{B} \sim v_{\mathrm{H}}$, since $v_{B}$ increases as the semimajor axis of the mutual binary orbit decreases. Therefore, we find from equation (14) that $\mathcal{R}_{\mathrm{sh}} / \mathcal{R}_{\mathrm{ex}} \sim v_{\mathrm{H}} / v \sim 10$ for our estimate of $v / v_{\mathrm{H}} \sim 0.1$. This implies that $\sim 10 \%$ of all binaries that formed will suffer an exchange reaction or break up. We performed numerical integrations of binary breakup and exchange reactions in order to obtain a more accurate estimate, and we find that only about $3 \%$ of the binaries suffer an exchange reaction and/or break up. Our order-of-magnitude calculation, therefore, slightly overestimates the number of binaries that experience an exchange reaction and/or break up. Moreover, only a fraction of the these binaries will end up as binaries with prograde orbits. In conclusion, we predict that the vast majority ( $\gtrsim 97 \%$ ) of binaries with comparable masses will have retrograde orbits if $\mathrm{KBO}$ velocities of $v \lesssim 0.1 v_{\mathrm{H}}$ prevailed during binary formation. This prediction assumes that subsequent orbital evolution due to dynamical friction does not alter the sense of the binary orbit after formation.

\subsection{Super-Hill Velocity: $v \gg v_{\mathrm{H}}$}

There is some uncertainty as to what the actual values of $\sigma$ and $\Sigma$ were during binary formation. For a few times larger value of $\Sigma$ and an unchanged value of $\sigma$, we enter the regime in which $v$ exceeds the Hill velocity (this can be seen from eq. [3]). We discuss here briefly how this would affect the ratio of prograde to retrograde binary orbits.
Schlichting \& Sari (2008) have shown that for $v \gg v_{\mathrm{H}}$, only binaries that form with a binary separation of $R_{\text {crit }}=R_{\mathrm{H}}\left(v_{\mathrm{H}} / v\right)^{2}$ or less tend to be saved from breakup. The $\mathrm{L}^{2} \mathrm{~s}$ mechanism fails to create binaries with separations of $\sim R_{\text {crit }}$ or less, since dynamical friction is not able to dissipate sufficient energy for tight binaries to form. Therefore, the $\mathrm{L}^{2} \mathrm{~s}$ mechanism is not important if the KBOs have super-Hill velocities. Tight binaries (with separations less than $\lesssim R_{\text {crit }}$ ) can form via the $\mathrm{L}^{3}$ mechanism. However, in this case, the binary formation cross section is significantly reduced with respect to the sub-Hill velocity regime (see Noll et al. [2008] and Schlichting \& Sari [2008] for details). The fact that retrograde orbits are stable for larger semimajor axes is no longer of importance, since only tight binaries tend to survive. We therefore predict that a roughly equal number of prograde and retrograde binaries form if super-Hill velocities prevail. This prediction is supported by Figure 4 . Figure 4 shows the ratio of retrograde binaries with a modified Jacobi constant of $J_{C}^{\mathrm{min}}$ or larger to the total number of binaries that formed via the $\mathrm{L}^{3}$ mechanism for $v \ll v_{\mathrm{H}}$. When all binaries are included, we find that about $2 / 3$ have retrograde orbits. More retrograde than prograde binaries form because retrograde binary orbits are stable further out in the Hill sphere than prograde ones. As $J_{C}^{\mathrm{min}}$ increases, the fraction of retrograde binaries decreases, reaching a minimum of about $1 / 3$ for $J_{C}^{\min } \sim 9$. This may be due to the Keplerian shear, which increases the duration of a prograde encounter between unbound KBOs compared to a retrograde encounter. The fraction of prograde and retrograde binaries becomes comparable for $J_{C}^{\mathrm{min}} \gg 9$, because for such binaries, neither the Keplerian shear nor the increased stability of retrograde orbits are important. This is the relevant regime for binaries that form for $v \gg v_{\mathrm{H}}$, since these large modified Jacobi constants correspond to tight binaries, which are the only binaries that are saved from breakup if super-Hill velocities prevail.

\section{COMPARISON WITH OBSERVATIONS}

To date, the orbits of more than a dozen KBBs have been well determined (e.g., Noll et al. 2008). Unfortunately, due to projection effects, the prograde and retrograde orbital solutions of the KBBs are nearly degenerate. This degeneracy can usually only be broken after several years, once the viewing angle of the KBBs has changed sufficiently. Very recently, after the submission of our original manuscript, two groups reported unique orbital solutions for the KBBs Typhon-Echidna (Grundy et al. 2008) and 2001 $\mathrm{QW}_{322}$ (Petit et al. 2008b). Grundy et al. (2008) find a prograde orbit for Typhon-Echidna, and Petit et al. (2008b) report a retrograde orbit for $2001 \mathrm{QW}_{322} .2001 \mathrm{QW}_{322}$ has such a large binary separation that, even in the current Kuiper Belt, it experiences significant dynamical interactions with other large KBOs. It is early to draw conclusions for the whole binary population, but if comparable numbers of retrograde and prograde binaries are found, this would imply that KBBs formed from a dispersiondominated KBO disk, which would also be consistent with observed binary inclinations. Dispersion-dominated KBO velocities would imply that the value of $\Sigma / \sigma$ was larger during binary formation than what we used in equation (3). However, the velocity dispersion during binary formation cannot have exceeded $v_{\mathrm{H}}$ significantly, since the binary formation timescales would otherwise become excessively long (Noll et al. 2008; Schlichting \& Sari 2008).

\section{DISCUSSION AND CONCLUSIONS}

The relative abundance of prograde to retrograde orbits enables us to differentiate between various proposed binary formation scenarios observationally. We predict that the vast majority $(\gtrsim 97 \%)$ of binaries with comparable masses will have retrograde orbits if 
KBO velocities of $\lesssim 0.1 v_{\mathrm{H}}$ prevailed during their formation. This dominance of retrograde over prograde binary orbits is due to the fact that for sub-Hill velocities, binaries form primarily via the $\mathrm{L}^{2} \mathrm{~S}$ mechanism, rather than the $\mathrm{L}^{3}$ mechanism. Since dynamical friction only gradually increases the modified Jacobi constant, all binaries that form via the $\mathrm{L}^{2} \mathrm{~s}$ mechanism, or any other mechanism that dissipates little energy over an orbital timescale, will start with modified Jacobi constants close to those of unbound KBOs. Only stable retrograde orbits exist for binaries with modified Jacobi constants similar to those of KBOs with initially circular orbits around the Sun that penetrate inside the Hill sphere. Therefore, KBBs have retrograde orbits, provided that they form from dynamically cold KBOs via the $\mathrm{L}^{2} \mathrm{~s}$ mechanism.

As the $\mathrm{KBO}$ velocities approach $v_{\mathrm{H}}$, the preference of retrograde orbits decreases. Further, we predict that a comparable number of prograde and retrograde binaries form for super-Hill KBO velocities. This is because only the $\mathrm{L}^{3}$ mechanism can form tight binaries that tend to survive if super-Hill velocities prevail (Schlichting $\&$ Sari 2008). The fact that retrograde orbits are stable for larger semimajor axes is no longer of importance, since only tight binaries tend to survive. This therefore leads to the formation of a roughly equal number of prograde and retrograde binaries for superHill KBO velocities.

The analysis presented here has also implications for some of the other proposed binary formation scenarios. Weidenschilling (2002) suggested that KBBs form by a collision among two KBOs inside the Hill sphere of a third. Although the $\mathrm{L}^{3}$ mechanism dominates over such a collisional binary formation scenario, we briefly discuss our predictions for this collisional binary formation mechanism. For sub-Hill velocities, more retrograde than prograde bi- naries form, because retrograde binary orbits are stable further out in the Hill sphere than prograde ones (i.e., the phase space for forming retrograde binaries is larger than that for prograde binaries). For super-Hill velocities, a comparable number of prograde and retrograde binaries form, because the fact that retrograde orbits are stable for larger semimajor axes is no longer of importance, since only tight binaries are saved from breakup. In the formation scenario of Astakhov et al. (2005), the existence of long-lived transient binaries that spend a long time in their mutual Hill sphere, near a periodic orbit, is responsible for the creation of KBBs. Lee et al. (2007) find an excess of prograde over retrograde binaries and suggest that this is a signature of their binary formation process. Our work indicates that an excess of prograde over retrograde binaries might simply be the result of the velocity regime (i.e., $v \sim v_{\mathrm{H}}$ ) in which the binaries form (see Fig. 4).

All of the above predictions rely on the assumption that subsequent orbital evolution due to dynamical friction and dynamical stirring of the Kuiper Belt does not alter the sense of the binary orbit. The Kuiper Belt has undergone a phase of dynamical excitation that probably modified the orbital properties of KBBs. A detailed study on how dynamical stirring of the Kuiper Belt and dynamical friction affects binary inclinations would be very worthwhile for determining whether these effects were able to reverse the binary orbit from prograde to retrograde rotation.

Some of the numerical calculations presented here were performed on Caltech's Division of Geological and Planetary Sciences Dell cluster. R. S. is an Alfred P. Sloan Fellow and a Packard Fellow. This research was partially supported by the ERC.
Astakhov, S. A., Lee, E. A., \& Farrelly, D. 2005, MNRAS, 360, 401

Cameron, A. G. W., \& Ward, W. R. 1976, Lunar Planet. Sci. Conf., 7, 120

Fraser, W. C., et al. 2008, Icarus, 195, 827

Fuentes, C. I., \& Holman, M. J. 2008, AJ, 136, 83

Funato, Y., Makino, J., Hut, P., Kokubo, E., \& Kinoshita, D. 2004, Nature, 427, 518

Goldreich, P., Lithwick, Y., \& Sari, R. 2002, Nature, 420, 643 2004, ARA\&A, 42, 549

Goldreich, P., \& Tremaine, S. 1980, ApJ, 241, 425

Grundy, W. M., et al. 2008, Icarus, 197, 260

Hamilton, D. P., \& Burns, J. A. 1991, Icarus, 92, 118

Hamilton, D. P., \& Krivov, A. V. 1997, Icarus, 128, 241

Hartmann, W. K., \& Davis, D. R. 1975, Icarus, 24, 504

Hayashi, C. 1981, Prog. Theor. Phys. Suppl., 70, 35

Hénon, M. 1970, A\&A, 9, 24

Hénon, M., \& Petit, J.-M. 1986, Celest. Mech., 38, 67

Hill, G. W. 1878, Am. J. Math., 1, 5

Ida, S. 1990, Icarus, 88,129

Innanen, K. A. 1979, AJ, 84, 960

Kenyon, S. J., \& Luu, J. X. 1999, AJ, 118, 1101

\section{REFERENCES}

Lee, E. A., Astakhov, S. A., \& Farrelly, D. 2007, MNRAS, 379, 229

McKinnon, W. B. 1989, ApJ, 344, L41

Noll, K. S., Grundy, W. M., Chiang, E. I., Margot, J.-L., \& Kern, S. D. 2008, in The Solar System Beyond Neptune, ed. M. A. Barucci et al. (Tucson: Univ. Arizona Press), 345

Petit, J.-M., \& Hénon, M. 1986, Icarus, 66, 536

Petit, J.-M., Kavelaares, J. J., Gladman, B. J., \& Laredo, T. 2008a, in The Solar System Beyond Neptune, ed. M. A. Barucci et al. (Tucson: Univ. Arizona Press), 71

Petit, J.-M., Kavelaares, J. J., Gladman, B. J., Margot, J.-L., Nicholson, P. D., Jones, R. L., Parker, J., \& Ashby, M. 2008b, in Asteroids, Comets, Meteors 2008 (Houston: LPI), http://www.lpi.usra.edu/meetings/acm2008/pdf/8354.pdf Rafikov, R. R. 2003, AJ, 125, 942

Schlichting, H. E., \& Sari, R. 2008, ApJ, 673, 1218

Trujillo, C. A., \& Brown, M. E. 2003, Earth Moon Planets, 92, 99

Trujillo, C. A., Jewitt, D. C., \& Luu, J. X. 2001, AJ, 122, 457

Weidenschilling, S. J. 2002, Icarus, 160, 212

Wetherill, G. W., \& Stewart, G. R. 1993, Icarus, 106, 190

Zhang, S.-P., \& Innanen, K. A. 1988, Icarus, 75, 105 